\title{
The insensitivity of schedule-induced polydipsia to conditioned taste aversions: Effect of amount consumed during conditioning
}

\author{
RICHARD L. HYSON and JULIE L. SICKEL \\ The American University, Washington, D.C. 20016 \\ PAUL J. KULKOSKY \\ Edward W. Bourne Behavioral Research Laboratory, Cornell Medical Center \\ White Plains, New York 10605 \\ and \\ ANTHONY L. RILEY \\ The American University, Washington, D.C. 20016
}

\begin{abstract}
In Experiment 1, animals poisoned following schedule-induced or prandial-induced saccharin consumption subsequently showed identical aversions to saccharin when tested under water deprivation. In Experiment 2, animals conditioned to avoid saccharin to similar levels under water deprivation were differentially affected when saccharin was subsequently presented on the baselines of schedule-induced and prandial-induced drinking. Together, these data indicate that the differential effects observed on schedule-induced and prandial-induced drinking when animals are poisoned following consumption under these two schedules do not reflect the differential acquisition of taste aversions, but instead reflect the differential tendencies to drink induced by the spaced and massed feedings.
\end{abstract}

In relation to drinking induced by water deprivation or the presentation of a single massed meal, scheduleinduced drinking (Falk, 1961) is insensitive to conditioned taste aversions; that is, the presentation of a previously poisoned saccharin solution only weakly and transiently suppresses schedule-induced polydipsia, an effect in marked contrast to the rapidly acquired and long-lasting aversions produced in water-deprived or mass-fed animals (Clarke \& Westbrook, 1978; Riley, Hyson, Baker, \& Kulkosky, 1980; Riley, Lotter, \& Kulkosky, 1979; Riley, Peele, Richard, \& Kulkosky, 1981; Roll, Schaeffer, \& Smith, 1969).

It has been suggested that this relative insensitivity of schedule-induced polydipsia (SIP) to food aver-

The authors would like to thank C. Baker and M. Ortuno for technical assistance and D. B. Peele for helpful comments on an earlier draft of this manuscript. R. L. Hyson is currently at the Department of Psychology, University of Colorado, Boulder, Colorado. J. L. Sickel is currently at the Department of Psychology, University of Maryland, College Park, Maryland. Requests for reprints should be sent to Anthony L. Riley, Department of Psychology, The American University, Washington, D.C. 20016, or to Paul J. Kulkosky, Edward W. Bourne Behavioral Research Lab, New York Hospital, Cornell Medical Center, Westchester Division, 21 Bloomingdale Road, White Plains, New York 10605. sions reflects a general tendency to drink following the spaced presentations of food under the scheduleinduced drinking procedure, a tendency sufficient to override a conditioned aversion to a previously poisoned solution (cf. Grote \& Brown, 1973; Riley \& Lovely, 1978). Given that spaced pellet deliveries are not within the water-deprivation or mass-feeding procedures, such a tendency would not be operating to override the occurrence of the conditioned aversion under these designs.

There is, however, at least one additional difference between SIP and drinking induced by water deprivation or massed feeding (prandial-induced) other than the spaced pellet delivery which could account for the differences in the effects conditioned aversions have on consumption induced by the various procedures. Under SIP, for example, subjects typically consume at asymptote approximately $25 \mathrm{ml}$ of water in a 1-h session on a variable-time 60-sec schedule of pellet delivery. Under $23.5 \mathrm{~h}$ water deprivation or following a mass meal of 60 pellets, subjects asymptotically consume, on the average, 12 and $5 \mathrm{ml}$ of water, respectively (see Riley et al., 1980; Riley et al., 1979). Following asymptotic drinking under each of these procedures, on-baseline conditioned taste aversion training is initiated. When 
animals are given access to the novel solution to be paired with toxicosis, rats under the various induction procedures consume different amounts of the solution as a result of the different baseline levels of fluid consumption. It is possible that the weak and transient taste aversions under SIP do not reflect an increased tendency to drink induced by pellet delivery, but, instead, reflect the acquisition of a weaker aversion due to the relatively large amounts of saccharin consumed during conditioning. While some investigators report no relation between amounts consumed during conditioning and testing (Archer \& Sjödén, 1979; Kalat, 1976), such a possibility is supported by Braveman and Crane (1977) and Deutsch (1978), who report that, while aversions are directly related to amount of solution consumed during conditioning, this relationship changes as the amount consumed exceeds $5 \mathrm{ml}$. Once this level is reached, aversions are inversely related to amount consumed; that is, weaker aversions are acquired with increase in levels consumed during conditioning. Although these reports suggest that aversions weaken as subjects consume larger amounts of the CS, neither of the aforementioned studies examined the high levels of consumption typically found with SIP. Given the apparent nonlinearity of amount consumed and subsequent aversion, it is possible that the inverse relationship is not maintained with such large amounts of consumption.

The present studies examined the contribution of the amount of solution consumed during conditioning to the relative insensitivity of SIP to conditioned taste aversions.

\section{EXPERIMENT 1}

If the insensitivity of SIP to conditioned taste aversions is a function of the rat's acquiring a weaker aversion under SIP than under prandial-induced drinking due to the large amounts of solution consumed during conditioning on the polydipsia baseline, then subjects conditioned under SIP should show weaker aversions than previously conditioned massfed subjects when both groups are subsequently tested under identical feeding conditions. If, however, the insensitivity of SIP to conditioned aversions is a function of the pellet-induced tendency to drink and not due to a differential acquisition of aversions, then both groups should show similar aversions when subsequently tested under identical feeding conditions. Experiment 1 tested these predictions.

\section{Method}

\section{Subjects}

Subjects were 24 experimentally naive, female rats of LongEvans descent, approximately 90 days of age at the beginning of the experiment. The subjects were maintained on a 12-h-light/ 12-h-dark cycle for the duration of the experiment.

\section{Apparatus}

The subjects were housed in individual wire-mesh cages. In the front of each cage were openings into which graduated Nalgene tubes were placed for presentations of water or $.1 \% \mathrm{w} / \mathrm{v}$ sodium saccharin (Fisher purified) during conditioned aversion training. Six BRS/LVE experimental chambers were used during polydipsia and prandial training. A $3-\mathrm{cm}$ hopper was located on the front wall $2 \mathrm{~cm}$ above the grid floor and $7 \mathrm{~cm}$ from the right side wall. A graduated Nalgene tube was located on the front wall $2 \mathrm{~cm}$ to the left of the food hopper. The spout of the tube protruded approximately $2 \mathrm{~cm}$ into the chamber and $3 \mathrm{~cm}$ above the grid floor.

\section{Procedure}

Phase 1: On-baseline aversion training. Initially, rats were deprived of food and given ad-lib access to water. Following reduction to $85 \%$ body weight, each subject was removed from its home cage and placed in the experimental chamber for a daily 1-h feeding session. For Group SIP $(n=12)$, a single $45-\mathrm{mg}$ Noyes pellet was delivered to each subject on a variable-time (VT) $60-\mathrm{sec}$ schedule until each subject had received a total of 60 pellets. For Group PD, 30 45-mg Noyes pellets were placed simultaneously in the food hopper for each subject at the beginning of each session. Five minutes later, 30 additional pellets were presented simultaneously, such that each subject received a total of 60 pellets in two massed meals. Water was continuously available to all subjects during both the spaced and massed feedings. Intakes were measured at the conclusion of the 1-h session. If necessary, supplemental feedings of Purina Rat Chow were given to subjects in the home cage $10 \mathrm{~min}$ after each daily session to maintain body weights at $85 \%$ of initial values. This procedure of daily 1-h sessions was continued for 25 consecutive days.

On Day 26, a novel saccharin solution replaced water during the daily free-food presentations. Fifteen minutes following this session, half of the subjects in each group (SIP-P and PD.P) were given an intraperitoneal (ip) injection of $1.8 \mathrm{mEq} / \mathrm{kg}, .15 \mathrm{M}$ $\mathrm{LiCl}$. The remaining subjects (Groups SIP-S and PD-S) were given an equivolume injection of distilled water $15 \mathrm{~min}$ following saccharin access.

Phase 2: Off-baseline extinction. For 2 days following the injection, all subjects were given ad-lib access to food and water in their home cages. At this point, water was removed and all subjects were given 20-min access to water daily for 3 consecutive days. On Day 1 of extinction, all subjects were given 20-min access to the previously poisoned saccharin solution in a onebottle test of the aversion to saccharin. On the following day, the subjects were given 20 -min access to water. This procedure of alternating saccharin and water presentations was continued until all subjects had received three extinction trials.

\section{Results}

All determinations of statistical significance were made at $\mathrm{p}<.05$, two-tailed.

\section{Phase 1: On-Baseline Aversion Training}

On Day 1 of water adaptation, the subjects in Group SIP drank approximately $5.3 \mathrm{ml}$ of water, which increased to approximately $18 \mathrm{ml}$ on Day 25 . The subjects in Group PD drank approximately $3.3 \mathrm{ml}$ on Day 1 and slightly more, $3.6 \mathrm{ml}$, on Day 25 . On Day 26, when saccharin was substituted for water during the feeding schedules, the subjects in both groups slightly increased saccharin consumption above the amount of water consumed on Day 25 , although this increase was significant only for Group 


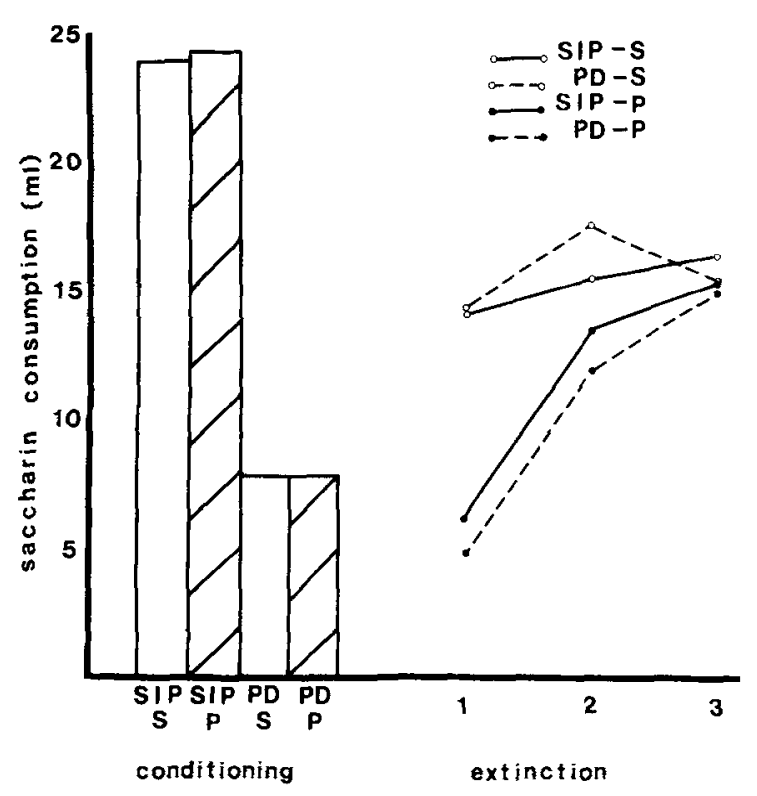

Figure 1. Mean consumption ( $\mathrm{ml}$ ) of saccharin for all groups during conditioning and extinction.

PD $[t(11)=1.35$ and $t(11)=2.27$ for Groups SIP and PD, respectively]. Group SIP drank significantly greater amounts of saccharin than Group PD on this initial exposure $[t(22)=4.37]$. Figure 1 (conditioning) presents the amount of saccharin consumed during conditioning for subjects in Groups SIP and PD.

\section{Phase 2: Off-Baseline Extinction}

When the water-deprived subjects were given 20-min access to water, there were no differences in the amount consumed among the four groups, each group consuming approximately $11 \mathrm{ml}$ on the first day of water adaptation. Over daily water exposure, all subjects gradually increased the amount consumed, drinking approximately $15 \mathrm{ml}$ on the final day of adaptation.

Figure 1 (extinction) presents the amount consumed when saccharin replaced water during the extinction sessions. On Day 1 of extinction, Groups SIP-P and PD-P drank significantly less saccharin than their respective controls, Groups SIP-S and PD-S $[t(10)=$ 2.63 and $t(10)=3.21]$. There were no significant differences in the amount of saccharin consumed between Groups SIP-P and PD-P or between Groups SIP-S and PD-S $[t(10)=.56$ and $t(10)=.01]$. Over extinction sessions, Groups SIP-P and PD-P increased consumption of saccharin, drinking amounts similar to those drunk by nonpoisoned subjects (SIP-S and PD-S) on the third and final extinction session. At no point in extinction were Groups SIP and PD significantly different.

\section{Discussion}

It is clear from these data that, whereas subjects consumed significantly different amounts of saccharin during conditioning under the schedule- and prandialinduced drinking procedures, all subjects displayed the same degree of aversion to saccharin when tested under the identical conditions of water deprivation. These data support the suggestion that, in relation to prandial-induced drinking, the relative insensitivity of SIP to conditioned taste aversions does not reflect the acquisition of weaker aversions due to the larger amounts of saccharin consumed during conditioning under polydipsia. That subjects conditioned under SIP showed aversions comparable to those of subjects trained under the massed feedings when tested under a situation without pellet deliveries suggests, further, that the aforementioned insensitivity reflects the pellet-induced tendency to drink under SIP (Riley et al., 1980; Riley et al., 1979).

While amount consumed during conditioning does not seem to be related to the degree of the aversion in subjects trained under different proceduresGroups SIP and PD displayed identical aversions, although the two groups drank different amounts during conditioning-significant differences appear within each group. As the amount consumed during conditioning increased for subjects within each poisoned group, the degree of the aversion, as indexed by extinction, decreased $[\mathrm{r}(4)=.853$ and .937 for Groups SIP-P and PD-P, respectively]. This correlation between amount consumed and aversions was not significant for the nonpoisoned subjects $[\mathrm{r}(4)=-.551$ and .563 for Groups SIP-S and PD-S, respectively].

Although the correlation between amount consumed and aversions was significant within each poisoned group, as described, the two poisoned groups did not differ in the degree of taste aversion displayed, despite the difference in the amount consumed during conditioning.

\section{EXPERIMENT 2}

In Experiment 1, it was demonstrated that the relative insensitivity of SIP to taste aversions was not a result of the acquisition of weaker aversions under the SIP procedure. As described earlier, this insensitivity may reflect an increased tendency to drink following pellet delivery.

In Experiment 2, animals were conditioned to avoid saccharin under identical conditions, that is, water deprivation. Following the acquisition of the taste aversion, the subjects were tested for the aversion under spaced (SIP) or massed (PD) pellet delivery. Any differences in the effect taste aversions have on 
SIP and PD after the subjects had acquired identical aversions under water deprivation must be attributable to differences in the pellet-induced tendency to drink under the two feeding schedules.

\section{Method}

\section{Subjects and Apparatus}

The subjects, 24 experimentally naive rats of the same sex, strain, and age as those used in the previous experiment, were maintained under the conditions described previously. The housing cages and experimental chambers were the same as those described in Experiment 1.

\section{Procedure}

Phase 1: Off-baseline aversion training. On Day 1, all subjects were deprived of water and given ad-lib access to food. On the next day, the subjects were given 20-min access to water in the home cage. This procedure was repeated for 10 consecutive days, at which point all subjects were approaching and drinking from the tube within $2 \mathrm{sec}$ of its presentation. On Day 11, differential treatment was administered to three groups of randomly selected subjects (Groups 4,2 , and 1 ). Group 4 was given 20 -min access to saccharin followed $15 \mathrm{~min}$ later by an ip injection of $1.8 \mathrm{mEq} / \mathrm{kg}$, $.15 \mathrm{M} \mathrm{LiCl}$. Groups 2 and 1 were given 20 -min access to water on this day. All subjects had 20-min access to water on the following day. On Day 13, Group 4 again received saccharin access followed by $\mathrm{LiCl}$. Groups 2 and 1 received access to water alone. Again, all subjects were given 20-min access to water on the following day. On Day 15, both Groups 4 and 2 received the saccharin- $\mathrm{LiCl}$ pairing. Group 1 continued to receive access to water. All subjects were given 20 -min access to water on the following day. Finally, on Day 17, Groups 4, 2, and 1 were poisoned following access to saccharin. In other words, different groups of subjects received 4,2 , or 1 saccharin- $\mathrm{LiCl}$ pairings, the final pairing for each group occurring on the same day (Day 17).

Phase 2: On-baseline extinction. On the day following the last conditioning trial, all subjects were given ad-lib access to food and water. Two days later, food was removed, and the subjects were reduced to $85 \%$ of ad-lib body weight. Following this reduction in body weight, each of the three groups of subjectsGroups 4, 2, and 1-was further divided into two groups, SIP and $P D$, and placed in the experimental chamber for a daily 1-h feeding session.

For Groups SIP-4, SIP-2, and SIP-1, a single 45-mg Noyes pellet was delivered to each subject on a VT 60 -sec schedule until each subject had received a total of 60 food pellets. For Groups PD-4, PD-2, and PD-1, 30 45-mg Noyes pellets were placed simultaneously in the food hopper for each subject at the beginning of the session. Five minutes later, 30 additional pellets were presented simultaneously, such that each subject received a total of 60 food pellets in two massed meals. Water was continuously available to all subjects during both the spaced and massed feedings. Intakes were measured at the conclusion of the 1 -h session. If necessary, supplemental feedings of Purina Rat Chow were given to the subjects in the home cage $10 \mathrm{~min}$ after each daily session to maintain body weights at $85 \%$ of initial values. This procedure of daily 1-h sessions was continued for 29 consecutive days.

On Day 1 of extinction, saccharin replaced water for all subjects during the feeding session. No injections were given following saccharin access during this extinction phase. On the following day, water was again available during the 1 -h session. This procedure of alternating saccharin/water presentations was continued until all subjects had received 10 extinction trials.

\section{Results}

All determinations of statistical significance were made at $\mathrm{p}<.05$, two-tailed.

\section{Phase 1: Off-Baseline Aversion Training}

Over the 10 water-adaptation days, rats in Groups 4,2 , and 1 increased consumption of water, drinking approximately $13 \mathrm{ml} /$ day over the final 3 days of water adaptation. When saccharin replaced water on Day 11, there was a slight, but nonsignificant, decrease in consumption from water baseline, each group drinking approximately $11 \mathrm{ml}$ of saccharin on this initial exposure.

Groups 4 and 2 significantly decreased consumption of saccharin over repeated saccharin- $\mathrm{LiCl}$ pairings, drinking approximately 0 and $4 \mathrm{ml}$ on the final saccharin exposure $[t(7)=7.70$ and $t(7)=5.66$ for Groups 4 and 2, respectively]. There was no offbaseline index of the aversion to saccharin for Group 1, subjects receiving only one saccharin- $\mathrm{LiCl}$ pairing.

\section{Phase 2: On-Baseline Extinction}

Schedule-induced polydipsia. On Day 1 of water adaptation, subjects in Groups SIP-4, SIP-2, and SIP-1 drank approximately $3 \mathrm{ml}$ of water, which increased to approximately $23 \mathrm{ml}$ on Day 29 . Figure 2 presents the amount of saccharin consumed for Groups SIP-4, SIP-2, and SIP-1, when saccharin replaced water on the polydipsia baseline. On this day, both Group SIP-4 and Group SIP-2 decreased consumption of saccharin below the previous day's water baseline $[\mathrm{t}(3)=4.02$ and $\mathrm{t}(3)=11.24$ for Groups SIP-4 and SIP-2, respectively], each group drinking approximately $0 \mathrm{ml}$ (see Figure 2, Day 1 of extinction). Group SIP-1 did not significantly decrease consumption of saccharin below the water baseline $[\mathrm{t}(3)=.69]$. While Groups SIP-4 and SIP-2 did not drink different amounts of saccharin on this exposure, both groups drank significantly less than Group SIP-1 $[\mathrm{t}(6)=4.51$ and $\mathrm{t}(6)=4.35$ for Groups SIP-4 and SIP-2, respectively].

With repeated exposures to saccharin, none of which was followed by poison, Group SIP-1 further increased saccharin consumption, drinking approximately $32 \mathrm{ml}$ on the second extinction trial. While

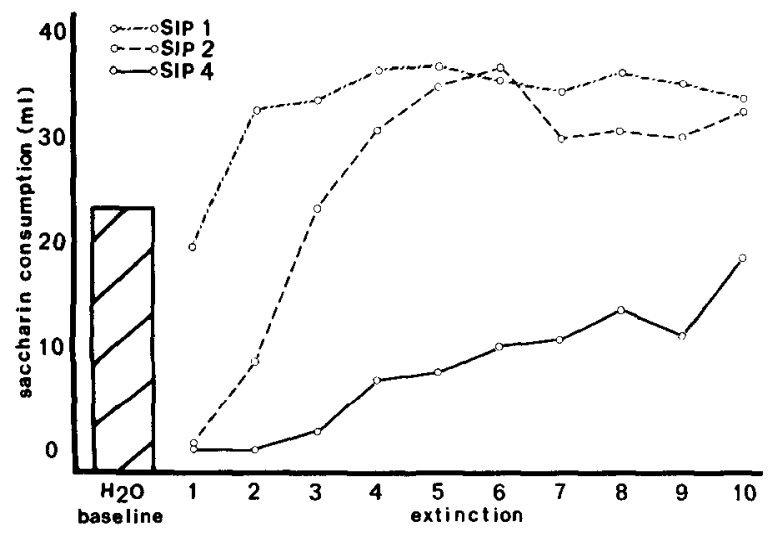

Figure 2. Mean consumption $(\mathrm{ml})$ of saccharin for Groups SIP-1, SIP-2, and SIP-4 over the multiple extinction trials following off-baseline conditioning. 
Group SIP-2 totally avoided saccharin on the first extinction trial, these subjects significantly increased consumption of saccharin $[t(3)=4.42]$ on the second extinction trial. Group SIP-2 further increased saccharin consumption over extinction, drinking approximately $34 \mathrm{ml}$ by the fifth extinction session. While Group SIP-4 totally avoided saccharin over the first three extinction sessions, these subjects did significantly increase consumption of saccharin by the sixth extinction trial. This group curve, however, does not accurately reflect individual subjects in Group SIP-4. Two subjects in this group never drank during extinction, whereas two subjects showed complete recovery, consuming levels similar to those of Group SIP-1 by the 6 th and 10 th extinction trial. On the final extinction test (Day 10), there were no significant differences in the amount of saccharin consumed among groups [all ts $(6)<1.12$ ].

Prandial-induced drinking. On Day 1 of water adaptation, subjects in Groups PD-4, PD-2, and PD-1 drank approximately $1.5 \mathrm{ml}$ of water, which increased to approximately $5 \mathrm{ml}$ on Day 29 . Figure 3 presents the amount of saccharin consumed for Groups PD-4, PD-2, and PD-1 when saccharin replaced water on the massed-feeding baseline. On this day, all groups decreased consumption of saccharin below the previous day's water baseline [all ts $(3)>3.92$ ] (see Figure 3, Day 1 of extinction). There were no differences among groups in the amount of saccharin consumed on this exposure [all ts $(6)<1.88$ ].

Group PD-1 significantly increased saccharin consumption by the third extinction trial and further increased consumption over repeated extinction sessions. There was no clear evidence, however, that asymptote was reached over these sessions. Although Group PD-2 totally avoided saccharin for five extinction trials, these subjects significantly increased saccharin consumption by Trial 9 . This group curve, again, does not reflect the individual subjects in Group PD-2 in that two subjects never drank saccharin, while two subjects significantly increased consumption by Trials 5 and 9 . While one subject

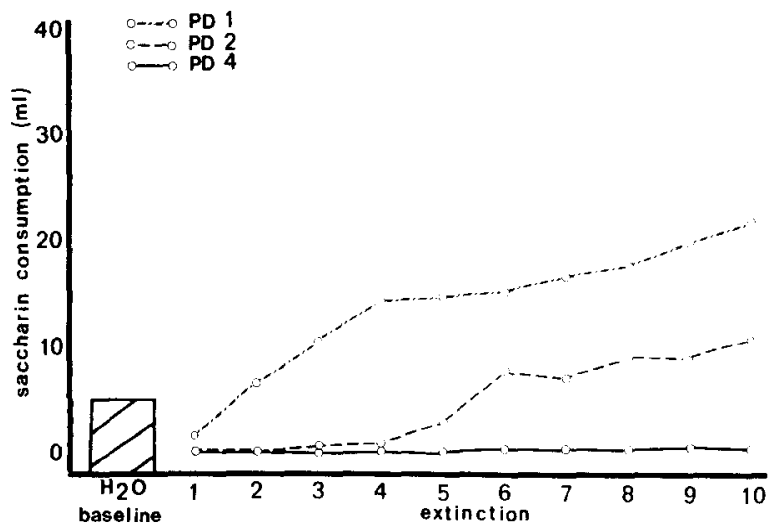

Figure 3. Mean consumption $(\mathrm{ml})$ of saccharin for Groups PD-1, PD-2, and PD-4 over the multiple extinction trials following off-baseline conditioning. in this group drank approximately $30 \mathrm{ml}$ over the last four extinction sessions, the remaining subjects and the group never recovered consumption to levels displayed by Group PD-1. Finally, Group PD-4 completely avoided saccharin throughout the entire extinction period. At no point did any subject consume more than $.50 \mathrm{ml}$ on any extinction trial. On the final extinction test (Day 10), Group PD-1 drank significantly more saccharin than both Group PD-2 and Group PD-4 [ $\mathrm{t}(6)=2.94]$. No other comparison was significant [all ts $(6)<.98$ ].

\section{Discussion}

Although subjects in both Group SIP and Group PD acquired identical aversions under water deprivation, the two groups were differentially sensitive to the presentation of the previously poisoned solution. While taste aversions disrupted consumption under both the schedule- and prandial-induced drinking procedures, drinking recovered faster and more completely for Group SIP. While the degree of the disruption in drinking was a function of the number of conditioning trials (cf. Nachman \& Hartley, 1974; Riley et al., 1980), the differences in the sensitivity of the two feeding schedules to taste aversions was evident at all levels of conditioning.

That SIP was less affected than prandial-induced drinking by taste aversions when both groups had acquired similar aversions is further support that the relative insensitivity of SIP does not reflect the differential acquisition of taste aversions, but instead is a function of the differential tendencies to drink induced by the feeding schedules.

\section{GENERAL DISCUSSION}

Experiments 1 and 2 indicate that the differences in the sensitivity of schedule-induced polydipsia and prandial-induced drinking reflect a performance difference and not a difference in learning between the two conditions (Riley et al., 1980). The insensitivity of SIP to taste aversions seems to be a result of the spaced pellet deliveries, which induce animals to drink. With each pellet delivery, the previously poisoned subjects sample the saccharin solution. Because the subjects are not poisoned following saccharin access during testing, the aversion to saccharin extinguishes. The interaction of the pelletinduced tendency to drink and the resulting extinction produces a faster and more complete recovery of drinking. While subjects in the prandial-induced drinking condition also extinguished the aversion to saccharin following sampling of the saccharin solution, the absence of the regular spaced pellet deliveries precluded the multiple and regular sampling of the previously poisoned solution; that is, there is no interaction of the induction to drink and extinction that characterizes Group SIP. 
It has been suggested (Freed, Zec, \& Mendelson, 1977; Riley et al., 1980; Riley et al., 1979) that the occurrence of schedule-induced alcohol polydipsia (Falk \& Samson, 1975; Falk \& Tang, 1977) is an index of the general insensitivity of SIP to conditioned taste aversions. This argument is based on the fact that although rats display SIP of alcohol, they will avoid the repeated consumption of intoxicating amounts of alcohol under ad-lib conditions in the home cage (Kulkosky, 1979; Kulkosky, Zellner, Hyson, \& Riley, 1980; Samson \& Falk, 1974). Apparently, the conditioned aversion to the taste of alcohol induced by the aversive effects of alcohol consumption, while sufficient to suppress home cage alcohol ingestion, does not affect schedule-induced alcohol polydipsia (Berman \& Cannon, 1974; Deutsch, Davis, \& Cap, 1976; Deutsch \& Eisner, 1977; Deutsch \& Walton, 1977; Deutsch, Walton, \& Thiel, 1978; Eckardt, 1975; Freed et al., 1977; Kulkosky, Sickel, \& Riley, 1980; Riley, Hyson, \& Kulkosky, Note 1).

Along with other work assessing the interaction of SIP and conditioned taste aversions (Clarke \& Westbrook, 1978; Riley et al., 1980; Riley et al., 1979; Roll et al., 1969), the present results support the position that SIP is insensitive to conditioned taste aversions, an insensitivity which can in part account for the occurrence of the schedule-induced consumption of alcohol.

\section{REFERENCE NOTE}

1. Riley, A., Hyson, R., \& Kulkosky, P. Alcohol-induced taste aversions: Mediation by the post-ingestional consequences of alcohol consumption. Unpublished manuscript available from authors upon request.

\section{REFERENCES}

Archer, T., \& Suödén, P. Positive correlation between pre- and postconditioning saccharin intake in taste-aversion learning. Animal Learning \& Behavior, 1979, 7, 144-148.

BARKer, L. M. CS duration, amount, and concentration effects in conditioning taste aversions. Learning and Motivation, 1976, 7, 265-273.

Berman, R. F., \& Cannon, D. S. The effect of prior ethanol experience on ethanol-induced saccharin aversions. Physiology \& Behavior, 1974, 12, 1041-1044.

Bond, N. W., \& DiGiusto, E. Amount of solution drunk is a factor in the establishment of taste aversion. Animal Learning \& Behavior, 1975, 3, 81-84.

Bond, N. W., \& Harland, W. Effect of amount of solution drunk on taste-aversion learning. Bulletin of the Psychonomic Society, 1975, 5, 219-220.

Braveman, N. S., \& Crane, J. Amount consumed and the formation of conditioned taste aversions. Behavioral Biology, $1977,21,470-477$.

Clarke, J. C., \& Westbrook, R. F. Control of polydipsic drinking by a taste aversion procedure. Pharmacology, Biochemistry, \& Behavior, 1978, 9, 283-286.

Deutsch, J., Davis, J., \& CAP, M. Conditioned taste aversions: Oral and postingestional factors. Behavioral Biology, 1976, $18,545-550$.

Deutsch, J., \& Eisner, A. Ethanol self-administration in the rat induced by forced drinking of ethanol. Behavioral Biology, $1977,20,81-90$.

Deutsch, J., \& W Alton, N. A rat alcoholism model in a free choice situation. Behavioral Biology, 1977, 19, 349-360.

Deutsch, J., Walton, N., \& Thiel, T. The importance of postingestional factors in limiting alcohol consumption in the rat. Behavioral Biology, 1978, 22, 128-131.

DEuTsCh, R. Effects of CS amount on conditioned taste aversion at different CS-US intervals. Animal Learning \& Behavior, $1978,6,258-260$.

EckardT, M. Conditioned taste aversion produced by the oral ingestion of ethanol in the rat. Physiological Psychology, 1975 , 3, 317-321.

FALK, J. L. Production of polydipsia in normal rats by an intermittent food schedule. Science, 1961, 133, 195-196.

FalK, J. L., \& Samson, H. H. Schedule-induced physical dependence on ethanol. Pharmacological Reviews, 1975, 27, 449-464.

FALK, J. L., \& TANG, M. Animal model of alcoholism: Critique and progress. In M. M. Gross (Ed.), Alcohol intoxication and withdrawal, IIIb: Studies in alcohol dependence. New York: Plenum Press, 1977.

Freed, W. J., Zec, R. F., \& Mendelson, J. Schedule-induced polydipsia: The role of orolingual factors and a new hypothesis. In J. A. W. M. Weijnen \& J. Mendelson (Eds.), Drinking behavior: Oral stimulation, reinforcement, and preference. New York: Plenum Press, 1977.

Grote, F. W., \& Brown, R. T. Deprivation level affects extinction of a conditioned taste aversion. Learning and Motivation, 1973, 4, 314-319.

KaLAT, J. W. Should taste-aversion learning experiments control duration or volume of drinking on the training day? Animal Learning \& Behavior, 1976, 4, 96-98.

Kulkosky, P. J. Effect of addition of ethanol and $\mathrm{NaCl}$ on saccharin + glucose polydipsia. Pharmacology, Biochemistry, \& Behavior, 1979, 10, 277-283.

Kulkosky, P. J., Sickel, J. L., \& Riley, A. L. Total avoidance of saccharin consumption by rats after repeatedly paired injections of ethanol of LiCl. Pharmacology, Biochemistry, \& Behavior, 1980, 13, 77-80.

Kulkosky, P. J., Zellner, D. A., Hyson, R. L., \& Riley, A. L. Ethanol consumption of rats in individual, group, and colonial housing conditions. Physiological Psychology, 1980, 8, 56-60.

Nachman, M., \& Hartley, P. L. Role of illness in producing learned taste aversions in rats: A comparison of several rodenticides. Journal of Comparative and Physiological Psychology, 1974, 86, 949-956.

Riley, A. L., Hyson, R. L., Baker, C. S., \& Kulkosky, P. J. The interaction of conditioned taste aversions and scheduleinduced polydipsia: Effects of repeated conditioning trials. Animal Learning \& Behavior, 1980, 8, 211-217.

Riley, A. L., LotTer, E. C., \& Kulkosky, P. J. The effects of conditioned taste aversions on the acquisition and maintenance of schedule-induced polydipsia. Animal Learning \& Behavior, 1979, 7, 3-12.

Riley, A. L., \& Lovely, R. H. Chlordiazepoxide-induced reversal of an amphetamine-established aversion: Dipsogenic effects. Physiological Psychology, 1978, 6, 488-492.

Riley, A. L., Peele, D. B., Richard, K. D., \& Kulkosky, P. J. The interaction of conditioned taste aversions and scheduleinduced polydipsia: Availability of alternative behaviors. Animal Learning \& Behavior, 1981, 9, 287-290.

Roll, D., Schaeffer, R. W., \& SMith, J. C. Effects of a conditioned taste aversion on schedule-induced polydipsia. Psychonomic Science, 1969, 16, 39-41.

Samson, H. H., \& Falk, J. L. Schedule-induced ethanol polydipsia: Enhancement by saccharin. Pharmacology, Biochemistry, \& Behavior, 1974, 2, 835-838.

(Received for publication August 14, 1980; revision accepted December 2, 1980.) 